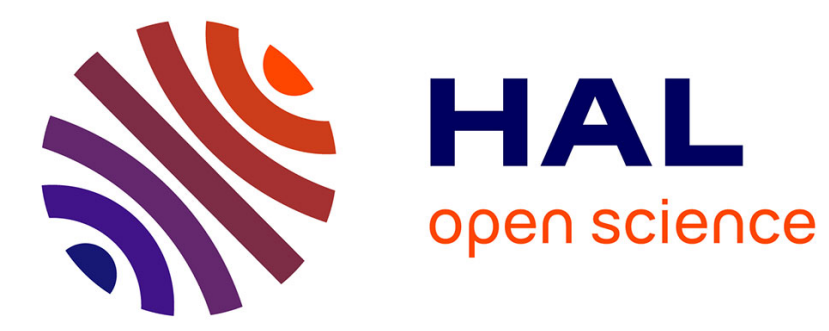

\title{
Reliable Fast Frequency Sweep for Microwave Devices via the Reduced Basis Method
}

\author{
Valentin de La Rubia, Ulrich Razafison, Yvon Maday
}

\section{To cite this version:}

Valentin de La Rubia, Ulrich Razafison, Yvon Maday. Reliable Fast Frequency Sweep for Microwave Devices via the Reduced Basis Method. IEEE Transactions on Microwave Theory and Techniques, 2009, 57 (12(1)), pp.2923-2937. 10.1109/TMTT.2009.2034208 . hal-00379198

\section{HAL Id: hal-00379198 \\ https://hal.science/hal-00379198}

Submitted on 27 Apr 2009

HAL is a multi-disciplinary open access archive for the deposit and dissemination of scientific research documents, whether they are published or not. The documents may come from teaching and research institutions in France or abroad, or from public or private research centers.
L'archive ouverte pluridisciplinaire HAL, est destinée au dépôt et à la diffusion de documents scientifiques de niveau recherche, publiés ou non, émanant des établissements d'enseignement et de recherche français ou étrangers, des laboratoires publics ou privés. 


\title{
Reliable Fast Frequency Sweep for Microwave Devices via the Reduced Basis Method
}

\author{
Valentín de la Rubia, Ulrich Razafison and Yvon Maday
}

\begin{abstract}
In this paper, a reduced basis approximation-based model order reduction for fast and reliable frequency sweep in the time-harmonic Maxwell's equations is detailed. Contrary to what one may expect by observing the frequency response of different microwave circuits, the electromagnetic field within these devices does not drastically vary as frequency changes in a band of interest. Thus, instead of using computationally inefficient, large dimension, numerical approximations such as finite element or boundary element methods for each frequency in the band, the point in here is to approximate the dynamics of the electromagnetic field itself as frequency changes. A much lower dimension, reduced basis approximation sorts this problem out. Not only rapid frequency evaluation of the reduced order model is carried out within this approach, but also special emphasis is placed on a fast determination of the error measure for each frequency in the band of interest. This certifies the accurate response of the reduced order model. The same scheme allows us, in an offline stage, to adaptively select the basis functions in the reduced basis approximation and automatically select the model order reduction process whenever a preestablished accuracy is required throughout the band of interest. Finally, real-life applications will illustrate the capabilities of this approach.
\end{abstract}

Keywords: Admittance matrix, computer aided engineering, design automation, error analysis, finite element methods, Galerkin method, reduced basis methods, reduced order systems.

\section{Introduction}

Current industrial needs are pushing microwave engineering to carry out even more complex electrical designs, requiring computer-aided design (CAD). Most numerical methods in electromagnetics were initially conceived merely as analysis tools due to their rather time-consuming characteristics. However, computational electromagnetics plays nowadays an important role on either qualified assistance for experienced engineers that are able to carry out an electrical design by means of trial and error, or global optimization techniques that automatically modify a given structure until some target electrical behaviour is achieved. The finite element method 
(FEM) is an appealing numerical method for its geometrical versatility and robutness, but its $\mathrm{CPU}$ time reduces its attraction whenever dense meshing and high frequency resolution over a wide band are required. However, two-dimensional (2-D) FEM analysis can reduce its rather time-consuming characteristics and makes it compatible with CAD [33]. Moreover, some threedimensional (3-D) FEM strategies can be taken into account and make certain CAD possible [32]. The use of adaptive mesh refinement may further alleviate dense mesh requirement. Another strategy lies in reduced order models as these may determine the frequency behaviour of a microwave device with ease. It is paradoxical that even though there are reduced order models for fast frequency sweep, rather conservative strategies, namely, time-consuming frequency point-wise analyses, are prefered for design automation. The rationale of this being in a lack of trust on reduced order models, especially in broadband applications.

The philosophy under model ordel reduction consists of replacing a rather complex mathematical model by a much simpler approximated one still maintaining certain aspects of the original model. This work concentrates on model order reduction for fast frequency sweep. Previous efforts on this issue in the electromagnetic community are mainly twofold: widespread momentmatching techniques and incoming singular-value decomposition (SVD) procedures. The former draw upon expanding the complex mathematical model into a Taylor series and building up an approximated model matching Taylor coefficients or moments. The latter starts with a large collection of field solutions at different frequencies. SVD is then applied to capture the essential dynamics of the original system by means of dominant singular value basis functions. A model of reduced complexity is finally obtained by Galerkin projection onto a subspace spanned by these basis functions.

On the one hand, asymptotic waveform evaluation (AWE) is proposed in [39]. AWE carries out a Padé approximation to increase the accuracy of a Taylor expansion whenever poles appear in the system response. However, its major drawback is the explicit computation of the moments to match. This results in numerical stagnation, since this procedure relies on a power method that quickly converges to the dominant eigenvector, giving rise to ill-conditioned projection matrices. Taking into account the same approximation strategy, a numerically stable Padé approximation via the Lanczos process is detailed in [15]. This result stems from the connection of the Lanczos process to Padé approximation, which states that the number of matching moments in the Lanczos algorithm is maximal. Further efforts for robust AWE are taken into account. [46] details how to orthogonalize the expansion vectors in the projection matrix and still match the moments in the system. This results in a well-conditioned reduced order model and prevents AWE from stagnation. In an attempt to increase the accuracy of momentmatching reduced order models over a wider frequency band, different expansion points are taken into account. Thus far, the new strategy is that each expansion vector not only preserves its own moment-matching properties, i.e., associated to its corresponding expansion point, but 
also captures the moments in every expansion point considered [44]. Critical aspects for appropriate model order reduction then arises: how many expansion points should be chosen, where to pick them, how many moments at each expansion point should be matched. [45] addresses these issues. An error estimate between the original system and its reduced order model for PVL-based techniques is proposed in [3] for single-input single-output systems, and extended for matrix-valued transfer functions in [4]. [42] uses PVL to carry out a fast frequency sweep in a domain decomposition framework. Further use of moment-matching approaches is taken into account in commercial software [48]. Notorious extension to multi-parameter configurations of these techniques is addressed in [14, 13], where not only a frequency sweep is carried out but also dielectric properties of different materials are swept. On the other hand, there is an increasing interest in SVD approaches. The determination of the dispersion diagram in inhomogeneous waveguides is addressed in [7]. Reduction of the large generalized eigenvalue problem to the subspace spanned by modal eigenvectors at evenly spaced frequency points and its derivates is considered. A similar strategy is taken into account for fast frequency sweep of microwave circuits in $[23,40]$. These works propose to adaptively select the number of sample points by explicitly computing the residual error at specific frequency points. This approach is further applied to domain decomposition in [24]. SVD techniques can take into account not only frequency as variable but also dielectric material properties and geometrical parameters. [1] addresses this in dielectric waveguide eigenanalysis. The major drawback of these techniques is that the number of sample points to achieve a given accuracy is unknown unless we explicitly compute the residual error in every single frequency to be evaluated. There are on-going efforts in this issue. Indeed, based on high frequency electromagnetics arguments, [47] points out an a priori estimate of the minimum number of sample points in scattering problems that, by nature, should be considered to provide proper results.

The reduced basis method was introduced in late 1970s for nonlinear structural analysis $[2,38]$. Since then, a large amount of developments has increased its efficiency and accuracy as well as spread out its range of applicability [6, 27, 5, 17, 28, 18, 43, 26, 37, 30, 36, 49, 29]. Previous reduced basis results in electromagnetics should be noticed. Rapid prediction of the radar cross section of conducting objects either in 2D or 3D problems is considered in $[22,21]$. Further efforts on efficient yet sharp a posteriori error bounds in reduced basis approximations for the Maxwell's system is addressed in [10], [51].

In this work, we propose a rapid and reliable frequency parameter sweep in microwave circuits based on the reduced basis method. This approach draws upon noting that the electromagnetic field does not arbitrarily vary as a function of frequency. On the contrary, it evolves on a very low dimensional subspace induced by the frequency variation. Instead of using a rather large dimensional finite element or boundary element approximation for every single frequency analysis, we change this approximation strategy to a much lower dimensional approximation 
framework - a reduced basis approximation. This concentrates on the evolution of the electromagnetic field as a function of the frequency parameter, rather than the approximation of the electromagnetic field itself earlier stated. Thus, we choose field solutions at different frequencies as the new approximation reduced basis. This settles down the new discretization framework. Galerkin procedure then reduces the large original system to a very low dimensional one, so the frequency response of the device under analysis can be obtained with ease. No miracle though, we still need to determine the new approximation basis (reduced basis) by some large dimension computations, but this is carried out only once. In this work, we pay special attention to decoupling those operations involving the large dimension of the original system from those computations in the many queries frequency evaluation stage of the reduced system. All time-consuming operations are carried out only once in the preliminary stage -offline. Once the reduced system is obtained, every single frequency evaluation has a negligible cost. Thus, we ensure a rapid frequency response evaluation. In addition, special emphasis is placed on a posteriori residual error determination. This certifies the accuracy of the response obtained in this approach. Contrary to previous efforts, the goal is to achieve a reduced order model where the numerical complexity of both, the frequency response evaluation and the a posteriori error estimate, no longer involves the large dimension of the original system. The same scheme allows us, in an offline stage, to adaptively select the basis functions in the reduced basis approximation and automatically select the model order reduction process whenever a preestablished accuracy is achieved throughout the band of interest.

This paper is organized as follows. In Section 2, we review the time-harmonic Maxwell's equations in variational form, establish the FEM formulation of the boundary value problem and define the frequency-parameterized variational problem that should be solved. Section 3 deals with the reduced basis approximation for the parameterized variational problem. A posteriori error considerations for reliable reduced basis approximations are discussed in Section 4. Section 5 sets out a generalized admittance matrix-based transfer function approach and its reduced basis counterpart that completely describes the electromagnetics in a given structure. Section 6 shows numerical examples and is conceived to illustrate the capabilities of the proposed approach as well as its accuracy. Finally, in Section 7, we comment on the conclusions.

\section{Problem Statement}

The time-harmonic Maxwell's equations can be written in a classical weak formulation over an appropriate admissible function space $\mathcal{X}$, viz.

$$
\begin{aligned}
& \text { Find } \bar{H} \in \mathcal{X} \text { such that } \\
& a(\bar{H}, \bar{w})=\varphi(\bar{w}) \quad \forall \bar{w} \in \mathcal{X}
\end{aligned}
$$


The bilinear form being

$$
a(\bar{H}, \bar{w})=\int_{\Omega}\left(\frac{1}{\varepsilon_{r}} \nabla \times \bar{H} \cdot \nabla \times \bar{w}-k^{2} \mu_{r} \bar{h} \cdot \bar{w}\right) d v,
$$

and the linear form $\varphi(\bar{w})=-\frac{j k}{\eta_{0}} \int_{\partial \Omega} \bar{\Phi} \cdot \bar{w} d s$, where $\varepsilon_{r}$ and $\mu_{r}$ are the relative permittivity and permeability of the medium respectively, $k=\omega \sqrt{\mu_{0} \varepsilon_{0}}$ is the wavenumber and $\eta_{0}=\sqrt{\mu_{0} / \varepsilon_{0}}$. $\Omega \subset \mathbb{R}^{3}$ is a source-free bounded domain. Here, the admissible space $\mathcal{X}$ represents $H($ curl; $\Omega)$ defined by:

$$
H(\operatorname{curl} ; \Omega)=\left\{\bar{h} \in\left(L^{2}(\Omega)\right)^{3} \mid \nabla \times \bar{h} \in\left(L^{2}(\Omega)\right)^{3}\right\} .
$$

We refer to [16] for a thorough explanation of this space. Let us denote as $\overline{n_{\Omega}}$ the unit outward normal vector on the boundary $\partial \Omega$ of $\Omega$, the boundary condition involves $\bar{\Phi}=\overline{n_{\Omega}} \times \bar{E}$ related to the tangential electric field on $\partial \Omega$ that is imposed as an excitation. Finally $\bar{E}$ and $\bar{H}$ are the electric and magnetic fields [19].

The solution to problem (2.1) can be approximated by means of the finite element method provided a tetrahedral mesh of the domain $\Omega$ is given and, for instance, a second order first family of Nédélec's elements are defined in the reference tetrahedron [35, 50]. A finite element space $\mathcal{X}^{\mathcal{N}}$ arises, approximating the solution space $\mathcal{X}$, thus

$$
\begin{gathered}
\text { Find } \bar{H}^{\mathcal{N}} \in \mathcal{X}^{\mathcal{N}} \text { such that } \\
a\left(\bar{H}^{\mathcal{N}}, \bar{w}\right)=\varphi(\bar{w}) \quad \forall \bar{w} \in \mathcal{X}^{\mathcal{N}}
\end{gathered}
$$

$\bar{H}^{\mathcal{N}}=\sum_{i=1}^{\mathcal{N}} h_{i} \bar{w}_{i}$, where $\left(\bar{w}_{i}\right)_{i}$ is the set of all finite element basis functions and $\mathbf{h}=\left(h_{i}\right)_{i}$ denotes the associated degrees of freedom of the discrete magnetic field. In order to obtain the field solution, a large sparse system of equations of $\operatorname{dimension} \mathcal{N} \equiv \operatorname{dim}\left(\mathcal{X}^{\mathcal{N}}\right)$ should be solved:

$$
\left(\mathbf{K}-k^{2} \mathbf{M}\right) \mathbf{h}=\varphi .
$$

It should be noted that system (2.5) is a straighforward translation of (2.4) into matrix form. If we denote

$$
\begin{gathered}
a^{K}(\bar{u}, \bar{w})=\int_{\Omega}\left(\frac{1}{\varepsilon_{r}} \nabla \times \bar{u} \cdot \nabla \times \bar{w}\right) d v \\
a^{M}(\bar{u}, \bar{w})=\int_{\Omega}\left(\mu_{r} \bar{u} \cdot \bar{w}\right) d v
\end{gathered},
$$

the matrices $\mathbf{K}$ and $\mathbf{M}$ are defined as follows: $K_{i j}=a^{K}\left(\overline{w_{i}}, \overline{w_{j}}\right)$ and $M_{i j}=a^{M}\left(\overline{w_{i}}, \overline{w_{j}}\right)$. Finally $\varphi$ denotes a vector of coefficients $\varphi_{i}=\varphi\left(\overline{w_{i}}\right)$. In the framework we are dealing with, where the frequency behaviour of a given device is to be determined, many FEM frequency analyses may result in a rather time-consuming task. Actually, the problem we aim at solving is the following:

$$
\begin{aligned}
& \text { Find } \bar{H}(k) \in \mathcal{X} \text { such that } \\
& a(\bar{H}(k), \bar{w} ; k)=\varphi(\bar{w} ; k) \quad \forall \bar{w} \in \mathcal{X}
\end{aligned}
$$

for every frequency point $k$ in a band of interest $\mathcal{D}$, where $\mathcal{D}$ is a bounded interval of the real axis. Throughout this work, we deliberately refer to the wavenumber $k$ as frequency. It should be 


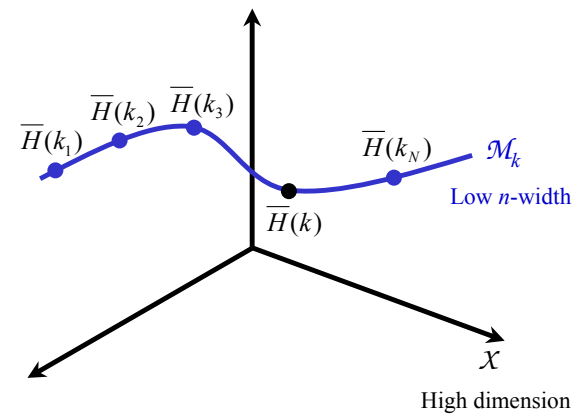

Figure 1: Low $n$-width set in which the magnetic field resides as frequency parameter changes.

noted that both, the bilinear form $a(\cdot, \cdot)$ and the linear functional $\varphi(\cdot)$, are modified as frequency changes. It is clear from the previous discussion that solving problem (2.7) by the finite element method may not be appropriate for every single frequency in the band.

\section{Reduced Basis Method}

The key feature upon which the reduced basis method stands is based on the following observation. The electromagnetic field in a given domain does not arbitrarily vary as a function of frequency, in other words, it is not an arbitrary element in $\mathcal{X}$. Instead, it evolves in a simple set induced by the frequency parameter $\mathcal{M}_{k}=\{\bar{H}(k), k \in \mathcal{D}\}$. In order to measure the complexity of the set of all solutions, the notion of $n$-width following Kolmogorov [20] can be of help (see also [27] for details in the reduced basis framework). This measures, for every integer $n$, the extent to which $\mathcal{M}_{k}$ may be approximated by a $n$-dimensional subspace of $\mathcal{X}$.

The key point in here is to provide an approximation of $\mathcal{M}_{k}$ instead of an approximation for an arbitrary member of $\mathcal{X}$, such as the finite element method proposes, since the field solution do not cover the entire space $\mathcal{X}$. Fig. 1 illustrates this situation.

\subsection{Reduced Basis Approximation}

By the preceeding arguments, the following space - reduced basis space- is proposed as an approximation of the set $\mathcal{M}_{k}$ of all solutions:

$$
\mathcal{W}^{N}=\operatorname{span}\left\{\bar{\zeta}_{1} \equiv \bar{H}\left(k_{1}\right), \ldots, \bar{\zeta}_{N} \equiv \bar{H}\left(k_{N}\right)\right\}
$$

The discretization proceeds by approximating the field solution $\bar{H}(k)$ at any frequency parameter by a Galerkin projection onto this space $\mathcal{W}^{N}$ spanned by few, well chosen solutions, viz.

$$
\begin{aligned}
& \text { Find } \widetilde{\bar{H}}(k) \in \mathcal{W}^{N} \text { such that } \\
& a(\widetilde{\bar{H}}(k), \bar{w} ; k)=\varphi(\bar{w} ; k) \quad \forall \bar{w} \in \mathcal{W}^{N}
\end{aligned}
$$


$\bar{H}(k) \simeq \widetilde{\bar{H}}(k) \equiv \sum_{i=1}^{N} \widetilde{h}_{i}(k) \bar{\zeta}_{i}$, where $\widetilde{\mathbf{h}}=\left(\widetilde{h}_{i}(k)\right)_{i}$ stands for the reduced basis approximation coefficient vector of the magnetic field. In order to obtain the reduced basis (3.8), we still need to rely on a finite element approximation, where large dimension $\mathcal{N}$ computations arise, but only at those $N$ selected frequency points, $k_{1}, \ldots, k_{N}$. Once this is carried out, the field solution at any frequency is obtained by solving a very small dense system of dimension $N \equiv \operatorname{dim}\left(\mathcal{W}^{N}\right)$, $(N \ll \mathcal{N})$ :

$$
\left(\widetilde{\mathbf{K}}-k^{2} \widetilde{\mathbf{M}}\right) \widetilde{\mathbf{h}}=\widetilde{\varphi}(k) .
$$

$\widetilde{\mathbf{K}}$ and $\widetilde{\mathbf{M}}$ matrices are given as follows: either $\widetilde{K}_{i j}=a^{K}\left(\overline{\zeta_{i}}, \overline{\zeta_{j}}\right)$ and $\widetilde{M}_{i j}=a^{M}\left(\overline{\zeta_{i}}, \overline{\zeta_{j}}\right)$, or $\widetilde{\mathbf{K}}=\mathbf{Z}^{T} \mathbf{K Z}$ and $\widetilde{\mathbf{M}}=\mathbf{Z}^{T} \mathbf{M Z}$, where $\mathbf{Z}$ is the matrix $\operatorname{col}\left\{\bar{\zeta}_{1}, \ldots, \bar{\zeta}_{N}\right\}$. Note that $Z$ is an $\mathcal{N} \times N$ matrix. $\widetilde{\varphi}$ denotes a vector of coefficients $\widetilde{\varphi}_{i}=\varphi\left(\overline{\zeta_{i}}\right)$, i.e., $\widetilde{\varphi}=\mathbf{Z}^{T} \varphi$.

The great advantage in the reduced basis approximation is that, even though $N \ll \mathcal{N}$, we are still able to achieve very good approximation results. The rationale of this being at approximating the evolution of the field as the parameter varies, rather than the field solution itself.

\subsection{Offline-Online Decomposition}

Even though solution to system (3.10) may seem straightforward to compute, there are some issues that should be addressed as regards the efficiency. In the reduced basis approach, we accept preliminary time-consuming computations, based on the finite element approximation. These are the construction of the reduced basis. But, once the reduced system is built, a fast computation is desired for the many frequency evaluations are to be carried out.

As is now standard, see $[37,49,29]$, we decompose the reduced basis procedure into two stages:

1. Off-line stage - the preprocessing stage which may be time-consuming is carried out only once. Here we can afford computations with complexity depending on $\mathcal{N}$, since they are just a few.

2. On-line stage - the other stage concerns the many queries frequency analysis stage and, therefore, we cannot afford any time-consuming operation online. We must remove any $\mathcal{N}$-contamination since only complexity depending on $N$ is desired in this stage.

Looking carefully at the reduced system (3.10) some questions arise. The system (3.10) can be solved in $O\left(N^{3}\right)$ operations, but what effort is required to assemble this system for a new frequency $k$ ? The left hand side can be assembled in $O\left(N^{2}\right)$ operations since the reduced stiffness and mass matrices $\widetilde{\mathbf{K}}, \widetilde{\mathbf{M}}$ can be computed offline and stored in memory. This is the result of an affine dependence on the frequency parameter of the bilinear form, i.e., it is a sum of products of frequency-dependent functions and frequency-independent bilinear forms, namely, 
$a\left(\overline{\zeta_{i}}, \overline{\zeta_{j}} ; k\right)=a^{K}\left(\overline{\zeta_{i}}, \overline{\zeta_{j}}\right)-k^{2} a^{M}\left(\overline{\zeta_{i}}, \overline{\zeta_{j}}\right)$. Concerning the right hand side, in all what follows, we make a simplifying assumption: taking into account homogeneous waveguide modal fields as excitation, we can actually know the frequency variation of the tangential electric field [42] and obtain an affine frequency dependency in the functional, viz.

$$
\varphi(\bar{w} ; k)=-\frac{j k}{\eta_{0}} g(k) \int_{\partial \Omega} \bar{\Phi}\left(k_{0}\right) \cdot \bar{w} d s=-\frac{j k}{\eta_{0}} g(k) f(\bar{w}),
$$

where $k_{0}$ is a specific frequency and

$$
g(k)= \begin{cases}1, & \text { for TEM and spherical modes } \\ \sqrt[4]{\frac{\left(\frac{k_{c_{i}}}{k_{0}}\right)^{2}-1}{\left(\frac{k_{c_{i}}}{k}\right)^{2}-1},} & \text { for TE modes } \\ \sqrt[4]{\frac{\left(\frac{k_{c_{i}}}{k}\right)^{2}-1}{\left(\frac{k_{c_{i}}}{k_{0}}\right)^{2}-1},} & \text { for TM modes }\end{cases}
$$

with $k_{c}$ being the cutoff frequency of the mode. This way, the right hand side in equation (3.10) $\widetilde{\varphi}(k)$ exhibits an offline-online decomposition that makes it possible to assemble it in $O(N)$ operations. Let us be more precise. $\widetilde{\varphi}(k)=\mathbf{Z}^{T} \varphi(k)$ and since $\varphi(k)$ has now an affine dependence on frequency, $\varphi(k)=-\frac{j k}{\eta_{0}} g(k) \mathbf{f}$ (see equation (3.11)), it can be computed as $\widetilde{\varphi}(k)=-\frac{j k}{\eta_{0}} g(k) \widetilde{\mathbf{f}}$, where $\tilde{\mathbf{f}}=\mathbf{Z}^{T} \mathbf{f}$ is carried out and stored offline only once.

In the more general case, when the previous assumption on the right hand side cannot be made, this contribution suffers from $\mathcal{N}$-contamination. Each time a new frequency $k$ is taken into account, $\widetilde{\varphi}(k)=\mathbf{Z}^{T} \varphi(k)$ has to be evaluated online involving $O(\mathcal{N} N)$ operations. The reason of this is that the functional $\varphi(\bar{w} ; k)=-\frac{j k}{\eta_{0}} \int_{\partial \Omega} \bar{\Phi}(k) \cdot \bar{w} d s$ shows a nonaffine parameter dependency in general. Recall that $\bar{\Phi}=\overline{n_{\Omega}} \times \bar{E}$ is related to the tangential electric field used as excitation. An alternative approximation has to be made in order to break the complexity also in this less favorable case. An affine parameter dependency can be invented via an empirical interpolation method $[5,17,28]$. We shall not elaborate on this in this paper and leave it for future work.

Although it may seem as we have removed all the numerical complexities depending on $\mathcal{N}$ in the online stage, note that there is still an underlying $\mathcal{N}$ dependency. It comes from the fact that the reduced basis functions themselves $\overline{\zeta_{i}}$ are written in terms of $\mathcal{N}$ finite element basis functions. Section 5.1 will deal with this in greater depth and completely remove the $\mathcal{N}$ dependency in the online stage.

Let us conclude this section by stating that, for the sake of accuracy in the numerical solution of the variational problem (3.9), an $\mathrm{H}_{\text {curl }}$-orthonormal basis of the reduced basis functions in $\mathcal{W}^{N}$ is actually used in the Galerkin procedure. Thus, (3.10) results in a better conditioned system of equations. This prevents from numerical stagnation 


\section{A Posteriori Error estimate}

The fundamental distinctive feature upon which this approach stands is discussed in this section. Once the reduced basis approximation is obtained, we carry out an a posteriori error estimate based on the residual error. This can certify whether a reduced basis solution is close enough to the actual field solution and thus ensures the reliability of the current approach. The goal in this work is that the residual error is computed with an operation count independent of $\mathcal{N}$, where only $O(N)$ operations arise $(N \ll \mathcal{N})$.

Let $\widetilde{\bar{H}}(k) \equiv \sum_{i=1}^{N} \widetilde{h}_{i}(k) \bar{\zeta}_{i}$ be the reduced basis approximation (see Subsection 3.1 ) to the actual field solution $\bar{H}(k)$, which we may be identified with the finite element field solution. The residual error $a(\bar{H}(k)-\widetilde{\bar{H}}(k), \bar{w} ; k)$ is then given as follows:

$$
\varphi(\bar{w} ; k)-a(\widetilde{\bar{H}}(k), \bar{w} ; k) \equiv \psi(\widetilde{\bar{H}}(k), \bar{w} ; k)
$$

for every $\bar{w}$ in $\mathcal{X}$. It should be noted that the residual error $\psi(\widetilde{\bar{H}}(k), \cdot ; k)$ is an element of $\mathcal{X}^{\prime}$, the dual space of $\mathcal{X} \equiv H(\operatorname{curl} ; \Omega)$. We quantify this residual by its norm:

$$
\|\psi(\tilde{\bar{H}}(k), \cdot ; k)\|_{\mathcal{X}^{\prime}}=\sup _{\bar{w} \in \mathcal{X}} \frac{|\psi(\tilde{\bar{H}}(k), \bar{w} ; k)|}{\|\bar{w}\|_{\mathcal{X}}}
$$

Taking advantage of fundamental results in functional analysis, since $\mathcal{X}$ is a Hilbert space with inner product $(\cdot, \cdot) \mathcal{X}$ and norm $\|\cdot\|_{\mathcal{X}}$, the Riesz Representation Theorem applies. Then, there exists a unique element $\bar{e}(k)$ in $\mathcal{X}$ such that

$$
(\bar{e}(k), \bar{w}) \mathcal{X}=\psi(\tilde{\bar{H}}(k), \bar{w} ; k) \quad \forall \bar{w} \in \mathcal{X}
$$

Furthermore, $\|\psi(\tilde{\bar{H}}(k), \cdot ; k)\|_{\mathcal{X}^{\prime}}=\|\bar{e}(k)\|_{\mathcal{X}}$. For a detailed explanation of dual spaces as well as the Riesz Representation Theorem we refer to [9].

Nevertheless, the residual error determination by means of either (4.14) or (4.15) lacks of practical interest. In both approaches the direct residual error operation count depends on $\mathcal{N}$ in the finite element context. With this constraint, only limited use of the a posteriori analysis can be performed, for very few frequencies, otherwise the global algorithm efficiency is compromised.

\subsection{Offline-Online Procedure}

The critical observation [26, 37] is that, under assumption (3.11), the right hand side of (4.15) shows an affine parameter dependency, i.e., it is a sum of products of frequency-dependent functions and frequency-independent functionals, viz.

$$
\begin{aligned}
& \psi(\widetilde{\widetilde{H}}(k), \bar{w} ; k)=-\frac{j k}{\eta_{0}} g(k) f(\bar{w}) \\
& -\sum_{i=1}^{N} \widetilde{h}_{i}(k) a^{K}\left(\overline{\zeta_{i}}, \bar{w}\right)+k^{2} \sum_{i=1}^{N} \widetilde{h}_{i}(k) a^{M}\left(\overline{\zeta_{i}}, \bar{w}\right)
\end{aligned}
$$


for every $\bar{w}$ in $\mathcal{X}$. Applying linear superposition to (4.15) we can write $\bar{e}(k)$ in $\mathcal{X}$ as:

$$
\bar{e}(k)=\frac{j k}{\eta_{0}} g(k) \overline{\mathcal{C}}+\sum_{i=1}^{N}{\widetilde{h_{i}}}_{(}(k) \overline{\mathcal{L}}_{i}^{K}-k^{2} \sum_{i=1}^{N} \widetilde{h}_{i}(k) \overline{\mathcal{L}}_{i}^{M}
$$

for $\overline{\mathcal{C}} \in \mathcal{X}$ satisfying $(\overline{\mathcal{C}}, \bar{w})_{\mathcal{X}}=-f(\bar{w}), \forall \bar{w} \in \mathcal{X}$, and $\overline{\mathcal{L}}_{i}^{p} \in \mathcal{X}$ satisfying $\left(\overline{\mathcal{L}}_{i}^{p}, \bar{w}\right)_{\mathcal{X}}=-a^{p}\left(\overline{\zeta_{i}}, \bar{w}\right)$, $\forall \bar{w} \in \mathcal{X}, i=1, \ldots, N, p \equiv K$ or $M$. Note that once again the Riesz Representation Theorem is applied. In this work, we use $(\bar{u}, \bar{w})_{\mathcal{X}}=\int_{\Omega}\left(\frac{1}{\varepsilon_{r}} \nabla \times \bar{u} \cdot(\nabla \times \bar{w})^{*}+\mu_{r} \bar{u} \cdot(\bar{w})^{*}\right) d v$ as inner product in $H_{\text {curl }}$, where $*$ denotes complex conjugate and $\varepsilon_{r}, \mu_{r}$ are assumed to be positive real values. Thus, we can concentrate on solving just a few finite element problems offline, namely,

$$
\begin{aligned}
(\mathbf{K}+\mathbf{M}) \mathbf{c} & =-\mathbf{f} \\
(\mathbf{K}+\mathbf{M}) \mathbf{l}_{\mathbf{i}}^{\mathbf{K}} & =-\mathbf{a}_{\mathbf{i}}^{\mathbf{K}} \\
(\mathbf{K}+\mathbf{M}) \mathbf{l}_{\mathbf{i}}^{\mathbf{M}} & =-\mathbf{a}_{\mathbf{i}}^{\mathbf{M}}
\end{aligned}
$$

and be ready to obtain $\bar{e}(k)$ at any frequency $k$ by a simple operation (see equation (4.17)). K and $\mathbf{M}$ are the FEM stiffness and mass matrices. $\mathbf{f}, \mathbf{a}_{\mathbf{i}}^{\mathbf{K}}$ and $\mathbf{a}_{\mathbf{i}}^{\mathbf{M}}$ stand for vectors of coefficients $f_{n}=f\left(\bar{w}_{n}\right), a_{i n}^{K}=a^{K}\left(\bar{\zeta}_{i}, \bar{w}_{n}\right)$ and $a_{i n}^{M}=a^{M}\left(\bar{\zeta}_{i}, \bar{w}_{n}\right)$, where $\overline{\zeta_{i}}$ is a reduced basis function. $\mathbf{c}, \mathbf{l}_{\mathbf{i}}^{\mathbf{K}}$ and $\mathbf{l}_{\mathbf{i}}^{\mathbf{M}}$ represent the coefficient vector of $\overline{\mathcal{C}}, \overline{\mathcal{L}}_{i}^{K}$ and $\overline{\mathcal{L}}_{i}^{M} . \overline{\mathcal{C}} \simeq \sum_{n=1}^{\mathcal{N}} c_{n} \bar{w}_{n}, \overline{\mathcal{L}}_{i}^{K} \simeq \sum_{n=1}^{\mathcal{N}} a_{i n}^{K} \bar{w}_{n}$ and $\overline{\mathcal{L}}_{i}^{M} \simeq \sum_{n=1}^{\mathcal{N}} a_{i n}^{M} \bar{w}_{n}$, where $\bar{w}_{n}$ is a finite element basis function. Note that problem (4.18) implies solving the same system of equations for different right hand sides. However, we are not interested on $\bar{e}(k)$ itself, but on its norm, which serves to quantify the residual error $\left(\|\bar{e}(k)\|_{\mathcal{X}}=\right.$ $\left.\|\psi(\widetilde{\bar{H}}(k), \cdot ; k)\|_{\mathcal{X}^{\prime}}\right)$. This norm can be readily obtained at any frequency $k$ simply by having computed the inner products $(\overline{\mathcal{C}}, \overline{\mathcal{C}})_{\mathcal{X}},\left(\overline{\mathcal{C}}, \overline{\mathcal{L}}_{i}^{p}\right)_{\mathcal{X}},\left(\overline{\mathcal{L}}_{i}^{p}, \overline{\mathcal{L}}_{i}^{q}\right)_{\mathcal{X}}, p, q \equiv K$ or $M, i=1, \ldots, N$, in the offline stage. This is the only information that must be stored from the offline stage. Thus,

$$
\begin{aligned}
& \|\bar{e}(k)\|_{\mathcal{X}}^{2}=\frac{k^{2}}{\eta_{0}^{2}}|g(k)|^{2}(\overline{\mathcal{C}}, \overline{\mathcal{C}})_{\mathcal{X}} \\
& +2 \operatorname{Re}\left[\frac{j k}{\eta_{0}} g(k) \sum_{i=1}^{N}\left(\widetilde{h_{i}}(k)\right)^{*}\left(\overline{\mathcal{C}}, \overline{\mathcal{L}}_{i}^{K}\right) \mathcal{X}\right] \\
& -2 k^{2} \operatorname{Re}\left[\frac{j k}{\eta_{0}} g(k) \sum_{i=1}^{N}\left(\widetilde{h_{i}}(k)\right)^{*}\left(\overline{\mathcal{C}}, \overline{\mathcal{L}}_{i}^{M}\right) \mathcal{X}\right] \\
& +\sum_{i=1}^{N} \widetilde{h_{i}}(k) \sum_{n=1}^{N}\left(\widetilde{h_{n}}(k)\right)^{*}\left(\overline{\mathcal{L}}_{i}^{K}, \overline{\mathcal{L}}_{n}^{K}\right) \mathcal{X} \\
& +k^{4} \sum_{i=1}^{N} \widetilde{h_{i}}(k) \sum_{n=1}^{N}\left(\widetilde{h_{n}}(k)\right)^{*}\left(\overline{\mathcal{L}}_{i}^{M}, \overline{\mathcal{L}}_{n}^{M}\right) \mathcal{X} \\
& -k^{2} \sum_{i=1}^{N} \widetilde{h_{i}}(k) \sum_{n=1}^{N}\left(\widetilde{h_{n}}(k)\right)^{*}\left\{\left(\overline{\mathcal{L}}_{i}^{K}, \overline{\mathcal{L}}_{n}^{M}\right) \mathcal{X}+\left(\left(\overline{\mathcal{L}}_{n}^{K}, \overline{\mathcal{L}}_{i}^{M}\right) \mathcal{X}\right)^{*}\right\}
\end{aligned}
$$

This makes it possible to promptly evaluate the residual error at any frequency $k$, just involving $O\left(N^{2}\right)$ operations. As previously stated, it is essential to obtain an online complexity independent of $\mathcal{N}$. Note that $\|\bar{e}(k)\|_{\mathcal{X}}^{2}=(\bar{e}(k), \bar{e}(k))_{\mathcal{X}}$. No miracle though, we still need to solve an additional system of equations in the offline stage. However, this strategy allows us to actually certify the wide band frequency response of the device so that we can completely rely on the reduced basis solution. This is crucial in a fast frequency sweep scheme, otherwise the reduced order model process is academic rather than of practical interest. 


\subsection{Adaptive Reduced Basis Selection: The Greedy Algorithm}

So far, we have not mentioned neither how many reduced basis functions are necessary to achieve a given accuracy nor how to choose them. A good strategy may be to choose field solutions at $N$ evenly spaced frequency points in the band of interest as reduced basis. However, we do not know a priori how many points should be selected to find a reasonable accuracy, although current efforts in this regard are taken into account [47]. As a result of our inexpensive a posteriori error estimate, we are now able to certify that the reduced order model is accurate enough throughout the band of interest, otherwise the dimension of the reduced basis should be increased to achieve a given accuracy. Nevertheless, we use a better strategy: an adaptive reduced basis selection. This adaptive selection is based on a greedy algorithm [49], [27]. Let $\left[k_{\min }, k_{\max }\right]$ be the frequency band of interest, and let $\mathcal{K}$ be a dense collection of frequency points in $\left[k_{\min }, k_{\max }\right]$ to recover the frequency response of the device under analysis in the band of interest. The following algorithm is proposed:

$$
\begin{aligned}
& \text { set } N=1 \\
& \text { choose } k_{1} \text { in } \mathcal{K} \text { randomly } \\
& \text { set } \mathcal{W}^{N}=\operatorname{span}\left\{\bar{H}\left(k_{1}\right)\right\} \\
& \text { while }\left(\max _{k \in \mathcal{K}}\|\psi(\widetilde{\bar{H}}(k), \cdot ; k)\|_{\mathcal{X}^{\prime}}>\epsilon_{\text {tol }}\right) \\
& \text { /*Residual not below a threshold throughout the band*/ } \\
& \text { choose } k_{N+1}=\arg \max _{k \in \mathcal{K}}\|\psi(\tilde{\bar{H}}(k), \cdot ; k)\|_{\mathcal{X}^{\prime}} \\
& \text { set } \mathcal{W}^{N+1}=\mathcal{W}^{N} \oplus \operatorname{span}\left\{\bar{H}\left(k_{N+1}\right)\right\} \\
& \text { set } N=N+1
\end{aligned}
$$

Note that this process requires no human interaction and the algorithm not only adaptively selects in some sense the best reduced basis functions but also automatically exits as soon as the preestablished accuracy $\epsilon_{t o l}$ is obtained throughout the whole frequency band $\left[k_{\min }, k_{\max }\right]$. Thus, we can completely rely on our reduced basis approximation.

\section{Generalized Admittance Matrix Approach}

Very often, the quantity of interest in the device under analysis is not the electromagnetic field itself, but an output that depends on the field solution. This section establishes an admittance matrix FEM formulation that completely describes the electromagnetics in the analysis domain [41]. For the sake of understanding, we take into account a specific radiating structure, namely, a wide band planar monopole. Fig. 2 shows the analysis domain $\Omega$. A coaxial port $\Gamma_{1}$ and a spherical port $\Gamma_{2}$ stand for the exciting and radiating ports respectively. It should be noted that spherical modal field expansion accounts for appropriate boundary truncation in the analysis 
domain. Therefore, a two-port network arises describing the electromagnetic behaviour in the analysis domain $\Omega$. As a result of the inclusion of the modal boundary conditions in problem (2.1), the tangential electric field on the modal ports may be written as a linear combination of waveguides modes $\overline{e_{t_{i}}}$ and $\overline{e_{t_{2}}}$. Thus,

$$
\begin{array}{ll}
\bar{\Phi}=\sum_{i=1}^{m_{1}} v_{1_{i}}\left(\bar{n} \times \overline{e_{t_{1_{i}}}}\right) & \text { on } \Gamma_{1} \\
\bar{\Phi}=\sum_{i=1}^{m_{2}} v_{2_{i}}\left(\bar{n} \times \overline{e_{t_{2_{i}}}}\right) & \text { on } \Gamma_{2}
\end{array}
$$

So far, the finite element discretization (2.5) turns into

$$
\left(\mathbf{K}-k^{2} \mathbf{M}\right) \mathbf{h}=-\frac{j k}{\eta_{0}}\left(\mathbf{B}_{\mathbf{1}} \mathbf{B}_{\mathbf{2}}\right)\left(\begin{array}{c}
\mathbf{v}_{\mathbf{1}} \\
\mathbf{v}_{\mathbf{2}}
\end{array}\right),
$$

where $\mathbf{v}_{\mathbf{1}}$ and $\mathbf{v}_{\mathbf{2}}$ are the coefficient vectors of the tangential electric field on $\Gamma_{1}$ and $\Gamma_{2}$, respectively. $\mathbf{B}_{\mathbf{1}}$ and $\mathbf{B}_{\mathbf{2}}$ are matrices with elements

$$
\begin{aligned}
& B_{1_{i j}}=\int_{\Gamma_{1}}\left(\bar{n} \times \overline{e_{t_{1_{j}}}}\right) \cdot \overline{w_{i}} d s \\
& B_{2_{i j}}=\int_{\Gamma_{2}}\left(\bar{n} \times \overline{e_{t_{2_{j}}}}\right) \cdot \overline{w_{i}} d s
\end{aligned} .
$$

Equation (5.21) determines the magnetic field $\bar{H}$ in the domain $\Omega$ in response to a superposition of modal tangential electric fields on the modal ports $\Gamma_{1}$ and $\Gamma_{2}$. Therefore, we know the tangential magnetic field on the modal ports and it can be established as a linear combination of modal tangential magnetic fields $\overline{h_{t_{i}}}$ and $\overline{h_{t_{2_{i}}}}$, viz.

$$
\begin{array}{ll}
\bar{n} \times \bar{H}=\bar{n} \times \sum_{i=1}^{m_{1}} i_{1_{i}} \overline{h_{t_{i}}} & \text { on } \Gamma_{1} . \\
\bar{n} \times \bar{H}=\bar{n} \times \sum_{i=1}^{m_{2}} i_{2_{i}} \overline{h_{t_{2_{i}}}} & \text { on } \Gamma_{2}
\end{array} .
$$

Working on this expression, the following integral relationship holds by using the finite element approximation of the magnetic field

$$
\begin{aligned}
& \sum_{i=1}^{\mathcal{N}} h_{i} \int_{\Gamma_{1}}\left(\bar{n} \times \overline{w_{i}}\right) \cdot \overline{e_{t_{1_{j}}}} d s=\sum_{i=1}^{m_{1}} i_{1_{i}} \int_{\Gamma_{1}}\left(\bar{n} \times \overline{h_{t_{1_{i}}}}\right) \cdot \overline{e_{t_{j}}} d s \\
& \sum_{i=1}^{\mathcal{N}} h_{i} \int_{\Gamma_{2}}\left(\bar{n} \times \overline{w_{i}}\right) \cdot \overline{e_{t_{2_{j}}}} d s=\sum_{i=1}^{m} i_{2_{i}} \int_{\Gamma_{2}}\left(\bar{n} \times \overline{h_{t_{2_{i}}}}\right) \cdot \overline{e_{t_{2_{j}}}} d s
\end{aligned},
$$

which in matrix form reads

$$
\begin{aligned}
& \mathbf{B}_{1}^{T} \mathbf{h}=\boldsymbol{\Delta}_{\mathbf{1}} \mathbf{i}_{\mathbf{1}} \\
& \mathbf{B}_{\mathbf{2}}^{T} \mathbf{h}=\boldsymbol{\Delta}_{\mathbf{2}} \mathbf{i}_{\mathbf{2}}
\end{aligned} .
$$

$\mathbf{i}_{\mathbf{1}}, \mathbf{i}_{\mathbf{2}}$ denoting the coefficient vectors of the modal tangential magnetic field on the modal ports and $\boldsymbol{\Delta}_{\mathbf{1}}, \boldsymbol{\Delta}_{\mathbf{2}}$ being diagonal matrices with elements

$$
\begin{aligned}
& \Delta_{1_{i j}}=\int_{\Gamma_{1}}\left(\bar{n} \times \overline{e_{t_{j}}}\right) \cdot \overline{h_{t_{1_{i}}}} d s, \quad i, j=1, \ldots, m_{1} . \\
& \Delta_{2_{i j}}=\int_{\Gamma_{2}}\left(\bar{n} \times \overline{e_{t_{j}}}\right) \cdot \overline{h_{t_{2_{i}}}} d s, \quad i, j=1, \ldots, m_{2} .
\end{aligned}
$$

Finally, manipulating (5.21) and (5.25) appropriately, a matrix relationship between the modal tangential electric and magnetic fields on the modal ports is obtained. Thus, 


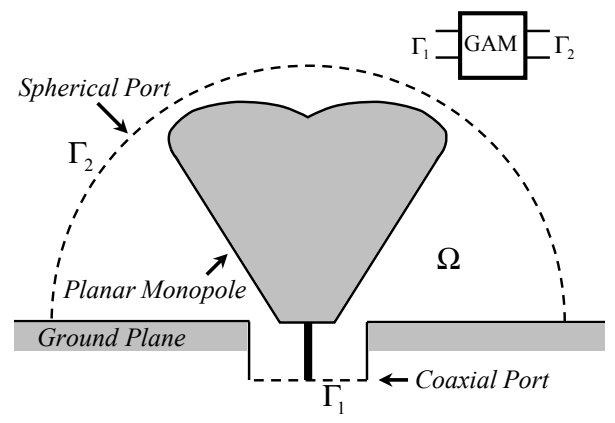

Figure 2: Wide band planar monopole antenna. Analysis domain and two-port network describing the electromagnetics in the antenna.

$$
\mathbf{i}=-\frac{j k}{\eta_{0}} \boldsymbol{\Delta}^{-\mathbf{1}} \mathbf{B}^{T}\left(\mathbf{K}-k^{2} \mathbf{M}\right)^{-1} \mathbf{B v}
$$

where $\mathbf{i}=\left(\mathbf{i}_{\mathbf{1}} \mathbf{i}_{\mathbf{2}}\right)^{T}, \mathbf{v}=\left(\mathbf{v}_{\mathbf{1}} \mathbf{v}_{\mathbf{2}}\right)^{T}, \mathbf{B}=\left(\mathbf{B}_{\mathbf{1}} \mathbf{B}_{\mathbf{2}}\right)$ and $\boldsymbol{\Delta}=\operatorname{diag}\left(\boldsymbol{\Delta}_{\mathbf{1}}, \boldsymbol{\Delta}_{\mathbf{2}}\right)$. Note that an amount of $m=m_{1}+m_{2}$ modes are taken into account. Insofar as a modal field normalization to obtain an identity $\boldsymbol{\Delta}$ matrix is accomplished, a symmetric $m \times m$ matrix relationship results.

$$
\mathbf{Y} \equiv-\frac{j k}{\eta_{0}} \mathbf{B}^{T}\left(\mathbf{K}-k^{2} \mathbf{M}\right)^{-1} \mathbf{B}
$$

This is the generalized admittance matrix (GAM). It should be noted that the GAM is a matrixvalued transfer function since it determines the system response to a given field excitation.

\subsection{Reduced Basis Approximation}

The problem we actually have to address is to obtain the GAM (5.28) for every single frequency in the band of interest. We set out the reduced basis approximation in this context. First of all, let us make all frequency dependence explicit in the GAM

$$
\mathbf{Y}(k)=-\frac{j k}{\eta_{0}} \mathbf{B}^{T}(k)\left(\mathbf{K}-k^{2} \mathbf{M}\right)^{-1} \mathbf{B}(k) .
$$

As previously stated (see Subsection 3.2), an analytical frequency variation arises in matrix B whenever homogeneous waveguides are taking into account [42]. Thus

$$
\mathbf{B}(k)=\mathbf{F} \mathbf{J}(k)
$$

with $\mathbf{F}=\mathbf{B}\left(k_{0}\right), k_{0}$ being a specific frequency and $\mathbf{J}(k)$ denoting a diagonal matrix with elements $(\mathbf{J}(k))_{i i}=g(k)$, where $g(k)$ is already defined in (3.12) for corresponding mode $i$. Equation (5.29) then reads

$$
\mathbf{Y}(k)=-\frac{j k}{\eta_{0}} \mathbf{J}^{T}(k) \mathbf{X}(k) \mathbf{J}(k)
$$


with

$$
\mathbf{X}(k)=\mathbf{F}^{T}\left(\mathbf{K}-k^{2} \mathbf{M}\right)^{-1} \mathbf{F} .
$$

We then concentrate in reducing system (5.32) via the reduced basis method instead of (5.31). The actual GAM $\mathbf{Y}(k)$ is straightforwardly obtained from the pseudo-GAM $\mathbf{X}(k)$ by means of analytical operations (5.31) [42].

In order to obtain the pseudo-GAM we should address the following finite element problem, which resembles solution to problem (2.4) for $m$ different excitations:

$$
\begin{aligned}
& \text { Find } \overline{\overline{\mathcal{H}}}(k) \in \mathcal{X}^{\mathcal{N}} \times \ldots{ }^{(m-1} \times \mathcal{X}^{\mathcal{N}} \text { such that } \\
& \mathcal{A}(\overline{\overline{\mathcal{H}}}(k), \overline{\overline{\mathcal{W}}} ; k)=\mathcal{F}(\overline{\overline{\mathcal{W}}}) \quad \forall \overline{\overline{\mathcal{W}}} \in \mathcal{X}^{\mathcal{N}} \times \ldots{ }^{(m-1} \times \mathcal{X}^{\mathcal{N}}
\end{aligned}
$$

where $\overline{\overline{\mathcal{H}}}(k)=\left(\bar{H}_{1}(k), \ldots, \bar{H}_{m}(k)\right)$ and $\overline{\overline{\mathcal{W}}}=\left(\bar{w}_{1}, \ldots, \bar{w}_{m}\right)$, the bilinear form $\mathcal{A}(\overline{\overline{\mathcal{H}}}(k), \overline{\overline{\mathcal{W}}} ; k)=$ $\sum_{i=1}^{m} a\left(\bar{H}_{i}(k), \bar{w}_{i} ; k\right)$ and the linear functional $\mathcal{F}(\overline{\overline{\mathcal{W}}} ; k)=\sum_{i=1}^{m} f_{i}\left(\bar{w}_{i}\right)$, with $f_{i}(\bar{w})=\int_{\partial \Omega}\left(\bar{n} \times \overline{e_{t_{i}}}\left(k_{0}\right)\right)$. $\bar{w} d s$. Note that this variational problem reads in matrix form either

$$
\operatorname{diag}\left(\mathbf{K}-k^{2} \mathbf{M}, \ldots{ }^{(m-1}, \mathbf{K}-k^{2} \mathbf{M}\right)\left(\begin{array}{c}
\mathbf{h}_{\mathbf{1}} \\
\vdots \\
\mathbf{h}_{\mathbf{m}}
\end{array}\right)=\left(\begin{array}{c}
\mathbf{f}_{\mathbf{1}} \\
\vdots \\
\mathbf{f}_{\mathbf{m}}
\end{array}\right)
$$

or

$$
\left(\mathbf{K}-k^{2} \mathbf{M}\right) \mathbf{H}=\mathbf{F},
$$

where $\mathbf{H}=\operatorname{col}\left\{\mathbf{h}_{\mathbf{1}}, \ldots, \mathbf{h}_{\mathbf{m}}\right\}$ and $\mathbf{F}=\operatorname{col}\left\{\mathbf{f}_{\mathbf{1}}, \ldots, \mathbf{f}_{\mathbf{m}}\right\}$ are $\mathcal{N} \times m$ matrices. $\mathbf{f}_{\mathbf{i}}$ denotes a vector of coefficients $f_{i_{j}}=f_{i}\left(\bar{w}_{j}\right)$ and $\mathbf{h}_{\mathbf{i}}$ represents the coefficient vector of the magnetic field for the $i$-th excitation problem. Therefore, in order to obtain the pseudo-GAM we just need to carry out

$$
\mathbf{X}(k)=\mathbf{F}^{T} \mathbf{H}(k) .
$$

Let us propose the following reduced basis approximation to problem (5.33).

$$
\begin{gathered}
\text { Find } \overline{\overline{\mathcal{H}}}(k) \in \mathcal{W}^{N} \text { such that } \\
\mathcal{A}(\overline{\overline{\mathcal{H}}}(k), \overline{\overline{\mathcal{W}}} ; k)=\mathcal{F}(\overline{\overline{\mathcal{W}}}) \quad \forall \overline{\overline{\mathcal{W}}} \in \mathcal{W}^{N} \quad,
\end{gathered}
$$

where we have chosen as reduced basis

$$
\mathcal{W}^{N}=\operatorname{span}\left\{\overline{\bar{\zeta}}_{1} \equiv \overline{\overline{\mathcal{H}}}\left(k_{1}\right), \ldots, \overline{\bar{\zeta}}_{N} \equiv \overline{\overline{\mathcal{H}}}\left(k_{N}\right)\right\}
$$

Then, $\overline{\overline{\mathcal{H}}}(k) \simeq \widetilde{\overline{\mathcal{H}}}(k) \equiv \sum_{i=1}^{N} \widetilde{h}_{i}(k) \overline{\bar{\zeta}}_{i}$. $\overline{\bar{\zeta}}_{i}$ being a reduced basis function and $\widetilde{h}_{i}(k)$ denoting its associated coefficient. As a result, the field solution for all the $m$ excitation problems is obtained at any frequency by solving a very small reduced system of dimension $N \equiv \operatorname{dim}\left(\mathcal{W}^{N}\right),(N \ll \mathcal{N})$ :

$$
\left(\widetilde{\mathcal{K}}-k^{2} \widetilde{\mathcal{M}}\right) \widetilde{\mathbf{h}}=\widetilde{\mathcal{F}}
$$


$\widetilde{\mathcal{K}}$ and $\widetilde{\mathcal{M}}$ matrices are given as follows: $\widetilde{\mathcal{K}}_{i j}=\mathcal{A}^{K}\left(\overline{\overline{\zeta_{i}}}, \overline{\overline{\zeta_{j}}}\right)$ and $\widetilde{\mathcal{M}}_{i j}=\mathcal{A}^{M}\left(\overline{\overline{\zeta_{i}}}, \overline{\overline{\zeta_{j}}}\right)$. Note that $\mathcal{A}\left(\overline{\overline{\zeta_{i}}}, \overline{\overline{\zeta_{j}}} ; k\right)=\mathcal{A}^{K}\left(\overline{\overline{\zeta_{i}}}, \overline{\overline{\zeta_{j}}}\right)-k^{2} \mathcal{A}^{M}\left(\overline{\overline{\zeta_{i}}}, \overline{\overline{\zeta_{j}}}\right)$. $\widetilde{\mathcal{F}}$ denotes a vector of coefficients $\widetilde{\mathcal{F}}_{i}=\mathcal{F}\left(\overline{\overline{\zeta_{i}}}\right)$, and $\widetilde{\mathbf{h}}$ stands for the reduced basis approximation coefficient vector. Remember that we still have all previouly developed reduced basis potential available, namely, offline-online decomposition, a posteriori error estimate and reduced basis construction via a greedy algorithm. The offlineonline decomposition deserves now further discussion. Recall that we had to deal with an underlying $\mathcal{N}$-contamination in the online stage in preceeding sections, since the reduced basis functions themselves are written in terms of $\mathcal{N}$ finite element basis functions. Here, we sort this problem out. Indeed, we are not interested on the field itself, but on the generalized admittance matrix describing the electromagnetics of the device under analysis. Once we have the reduced basis approximation for the multiple excitation problem $\overline{\overline{\mathcal{H}}}(k) \simeq \sum_{i=1}^{N} \widetilde{h}_{i}(k) \overline{\bar{\zeta}}_{i}$, the pseudo-GAM then reads

$$
\mathbf{X}(k) \simeq \sum_{i=1}^{N} \widetilde{h_{i}}(k) \mathbf{X}_{\mathbf{i}}
$$

$\mathbf{X}_{\mathbf{i}}=\mathbf{F}^{T} \mathbf{H}\left(k_{i}\right)$ are the pseudo-GAM ( $m \times m$ matrices $)$ at those frequency points used to obtain the reduced basis functions. Recall $\overline{\bar{\zeta}}_{i} \equiv \overline{\overline{\mathcal{H}}}\left(k_{i}\right)$ and see (5.36). Thus, we only need to compute and store $\mathbf{X}_{\mathbf{i}}$ offline to remove all $\mathcal{N}$ dependence in the online stage. Insofar as the pseudo-GAM at any frequency point is ready, the GAM is straightforwardly computed (5.31).

\section{$6 \quad$ Numerical Results}

In this section we apply the proposed model order reduction methodology to different challenging structures, namely, a dual-mode circular waveguide filter, a cylindrical dielectric resonator filter and band-notched planar monopole antennas. The capabilities and realiability of the proposed technique will be apparent throughout these examples. All computations were carried out on a 64-bit workstation with $2.00 \mathrm{GHz}$ Intel E5405 processor and 8 GB RAM.

\subsection{Dual-Mode Circular Waveguide Filter}

We take into account a four-pole elliptic dual-mode circular cavity filter in this example. Fig. 3 shows the filter setout as well as its dimensions. The two circular cavities are connected to corresponding input and output WR75 rectangular waveguides through identical slots. Furthermore, both cavities are connected by a cross-shaped iris. Finally, each cavity has got a horizontal tunning screw and a $\pi / 4$ tilt coupling screw. Although two physical cavities are considered, two degenerated modes are excited in each cavity. This gives rise to four electrical cavities reducing filter size and weight.

The electrical specifications of the filter are as follows: better than $20 \mathrm{~dB}$ return losses in a bandwidth of $100 \mathrm{MHz}$ centred at $11.8 \mathrm{GHz}$ and better than $15 \mathrm{~dB}$ out of band rejection. This 
filter was designed in [34] by means of mode-matching method and was further studied in [12] in a domain decomposition method framework. Instead of analyzing the whole analysis domain, we carried out the same modal domain decomposition strategy carried out in [12]: the analysis domain is decomposed into subdomains according the availability of modal field description [41]. This gives rise to analytical subdomains (circular waveguide sections) and subdomains where FEM resolution is mandatory. Fig. 4 illustrates this scheme. Since both input and output rectangular waveguide transitions to circular waveguide (transitions 1 and 2 in Fig. 4) are identical, it is only necessary to analyze one of these. A reduced basis-based reliable fast frequency sweep is taken into account in corresponding building blocks. Fig. 5 compares the results of this approach with measurements in [34]. Good agreement is achieved. Note that we carry out two overall model order reduction processes. On the one hand, we are just interested in the filter band response. Thus, we specify the $11.6-12 \mathrm{GHz}$ band as the band of interest to our fast frequency sweep algorithm. As previously stated, the algorihm itself adaptively selects the reduced basis functions based on a greedy strategy. This minimizes the residual error of the reduced basis approximation at every single frequency in the band of interest. Fig. 6(a) shows the residual error behaviour in the rectangular to circular waveguide transition subdomain as the reduced basis approximation order increases in the greedy framework. Similar results are obtained in the remaining building blocks. This procedure goes on until a preestablished error tolerance is achieved throughout the whole frequency band. This gives rise to a reliable reduced order model. Note that the residual error as well as the reduced solution at any frequency are easily computed due to the offline-online decomposition strategy. This makes it possible to compute the residual error and reduced solution for all frequencies in $O\left(N^{2}\right)$ and $O\left(N^{3}\right)$ operations each, respectively, where $N$ is the dimension of the reduced basis approximation. On the other hand, we are interested in the wide band response of the filter. Then, we specify the 10-15 GHz band as the band of interest to our fast frequency sweep. Once again, our algorithm adaptively chooses the reduced basis functions until the residual error throughout the band goes under a preestablished threshold. Fig. 6(b) details the residual error behaviour over the band of interest as the dimension of the reduced basis approximation increases. We emphasize that a reliable filter band response is also ensured in the wide band analysis. Nevertheless, a larger reduced basis dimension $N$ is required this time since not only the filter band is of interest but also the out of band filter rejection. Table 1 summarizes the final dimension of the reduced basis approximation in corresponding analysis subdomains within each approximation context, namely, filter band and wide band responses.

There is still an issue that deserves further discussion. Looking carefully at Fig. 6(b), two residual error peaks are noticed around 12.2 and $13 \mathrm{GHz}$. These belongs to interior resonances in the analysis subdomain. Indeed, the formulation we are using in this work is deliberately an Ewall formulation, thus there are some forbidden frequencies at which the FEM matrix $\mathbf{K}-k^{2} \mathbf{M}$ 


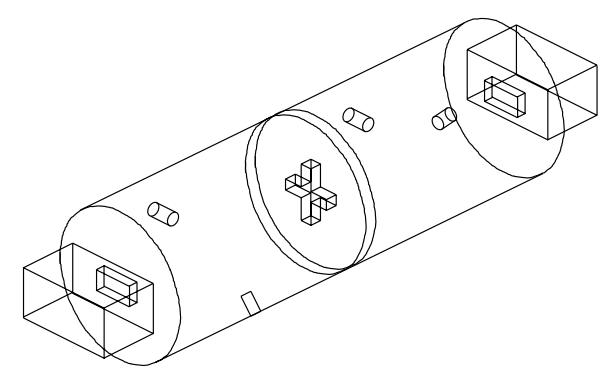

Figure 3: Dual-mode circular waveguide filter geometry. Cavity length $43.87 \mathrm{~mm}$, radius $14 \mathrm{~mm}$, iris thicknesses $1.5 \mathrm{~mm}$, slot lengths $10.05 \mathrm{~mm}$, slot widths $3 \mathrm{~mm}$, arm widths $2 \mathrm{~mm}$, horizontal arm length $7.65 \mathrm{~mm}$, vertical arm length $8.75 \mathrm{~mm}$, tunning screw depth $3.66 \mathrm{~mm}$ and coupling screw depth $3.35 \mathrm{~mm}$.

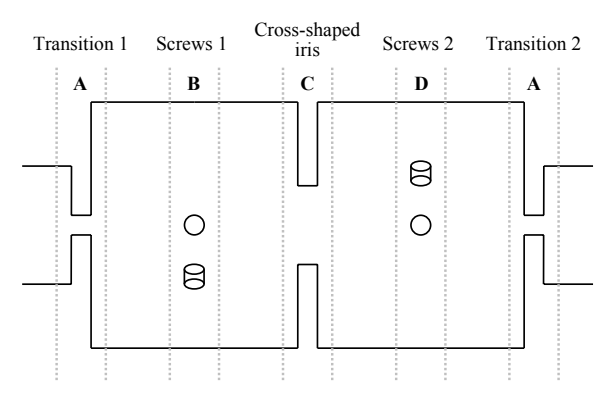

Figure 4: Side view of the dual-mode circular waveguide filter. Modal domain decomposition framework.

is singular. As a result, interior resonances do appear in the generalized admittance matrix approach. It should be noticed that the smaller the analysis domain is the higher these interior resonances are. The interior resonance problem can be sorted out by inclusion of dissipative radiation conditions in the formulation, which ensures solvability of the Maxwell's system at any frequency. In this work, we get rid of the interior resonance contanimation in the residual error by neglecting the residual error in a neighbourhood of each reduced basis sample point, where it is assumed to be small. Thus far, interior resonance residual error peaks have no effect on the greedy algorithm apart from forcing sampling around interior resonance frequencies whenever required.

\subsection{Cylindrical Dieletric Resonator Filter}

In this example, we address a coaxial-fed dielectric resonator filter made up of two cylindrical dielectric resonators with a concentric cylindrical hole. The geometry of this structure is shown in Fig. 7. This filter was considered in [8], where all dimensions and materials can be found, and was designed to perform a bandstop from 5 to $6.8 \mathrm{GHz}$. This time we take into account the 


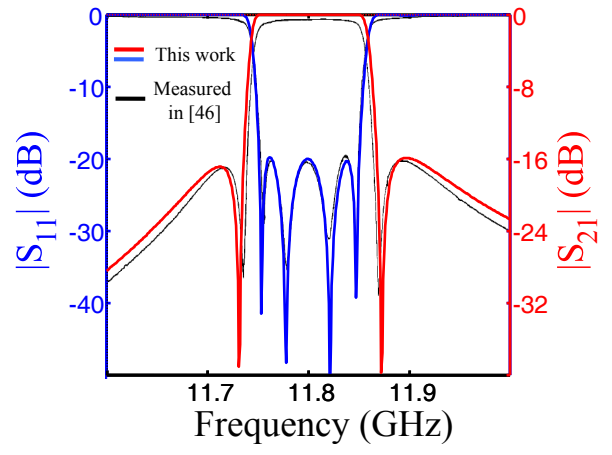

(a)

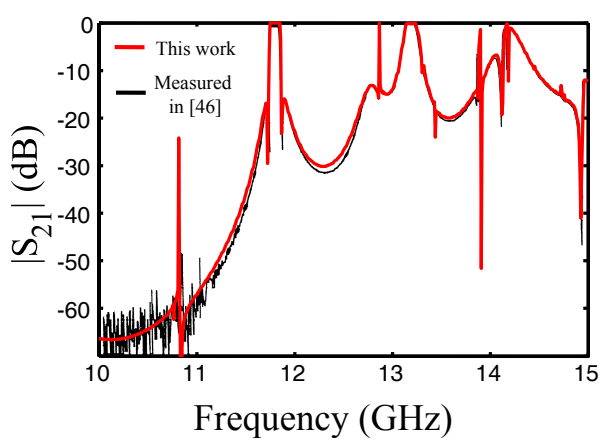

(b)

Figure 5: Dual-mode circular waveguide filter reduced order model results are compared with measurements. (a) Filter Band. (b) Wide band response.

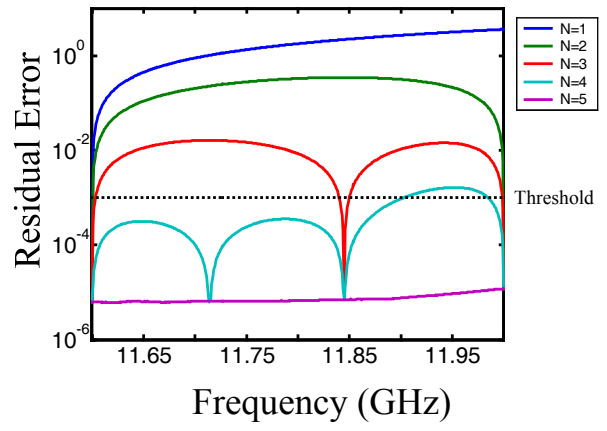

(a)

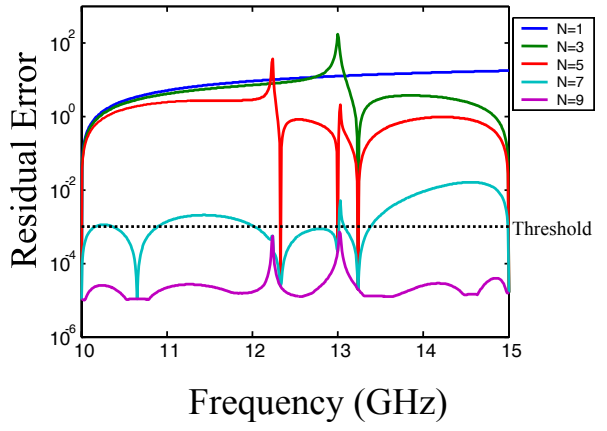

(b)

Figure 6: Evolution of the residual error in the rectangular to circular waveguide transition subdomain in the dual-mode filter analysis as a result of the greedy algorithm. (a) Filter band. (b) Wide band.

Table 1: Dimension of the Reduced Basis Approximation in the Dual-Mode Circular Waveguide Filter

\begin{tabular}{c|c|c}
\hline \multirow{2}{*}{ Subdomain } & \multicolumn{2}{|c}{ Reduced Basis Dimension $N$} \\
\cline { 2 - 3 } & $11.6-12 \mathrm{GHz}$ band & 10-15 GHz band \\
\hline \hline Transitions 1 and 2 & 5 & 9 \\
Cross-shaped Iris & 5 & 14 \\
Screws 1 & 5 & 14 \\
Screws 2 & 5 & 14 \\
\hline
\end{tabular}




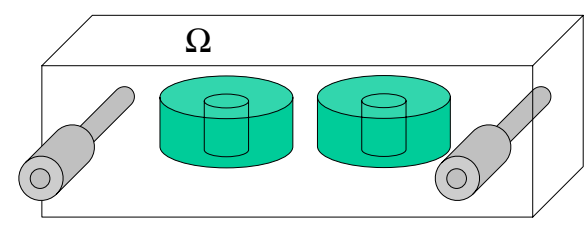

Figure 7: Geometry of the cylindrical dielectric resonator filter.

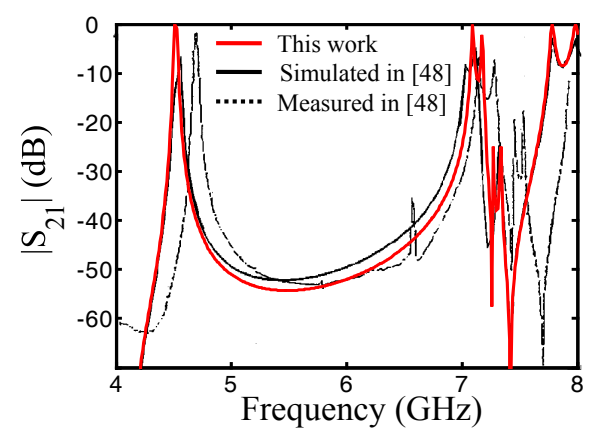

Figure 8: Cylindrical dielectric resonator filter reduced order model results are compared with measurements.

whole filter as analysis domain and apply our fast frequency sweep in the 4-8 GHz band. Fig. 8 compares the measurements and FEM simulation carried out in [8] with current formulation results for the transmission coefficient. Reasonable agreement is obtained. Fig. 9 shows the evolution of the residual error throughout the band of interest. On this occasion, we need a reduced order model of dimension $N=17$ until good results are certified in the whole band. Note that frequency sample points as well as the dimension of the reduced basis approximation are adaptively selected and no human interaction is required apart from the specification of the band of interest. Furthermore, the fast frequency sweep automatically exits whenever a realiable frequency response is obtained throughout the band of interest.

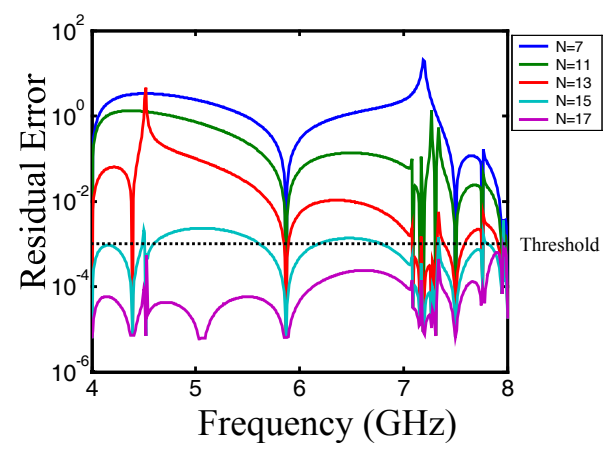

Figure 9: Greedy algorithm-based residual error evolution in the construction of the reduced basis functions for the cylindrical dielectric resonator filter. 


\subsection{Band-notched Planar Monopole Antennas}

Finally, we deal with different band-notched wide band planar monopole antennas. On the one hand, we take into account a wide band planar monopole where multiple band rejection is allowed. Thus, a multiband radiating structure arises rejecting inteference from narrowband communication systems as well as preventing interference to them. Fig. 10 details the radiating structure as well as its dimensions. Both $\cup$ and $\cap$-shaped slots are symmetrically inserted in the wide band planar monopole, giving rise to two notch filters. These slots avoid any radiating mode on the planar monopole at frequencies where their length becomes half a wavelength. This multiple band-notched monopole is addressed in [25]. We carry out a fast frequency sweep analysis with the proposed approach in the 1-7 GHz band. A reduced order model of dimension $N=15$ is required to provide a reliable response throughout the whole band. In addition, we carry out an FEM analysis at 15 evenly spaced frequency points in the band of interest. Fig. 11 compares the reflection coefficient results with measurements carried out in [25], as well as details the reduced basis adaptive sample points in the frequency axis. Note that some antenna dimensions are not provided in [25]. Reasonable agreement is found. It should be noted that the FEM analysis does not account for the sharp frequency notches in the antenna response.

On the other hand, we deal with an ultra wide band (UWB) antenna with band-rejection requirements. Typically, UWB systems (2-11 GHz band) should coexist with other services such as WLAN systems (5-6 GHz band). Here, we analyze an UWB antenna with WLAN band

rejection shown in Fig. 12. Based on the topology characteristics of the previous radiating structure, this antenna was designed in [31], where all dimensions are detailed. An profileoptimized UWB planar monopole antena was initially considered [32] and conformal-shaped slots were then optimized by a genetic algorithm within the methodology proposed in [11]. So far, a good rejection in the WLAN band is achieved while keeping good radiation characteristics in the remaining UWB band. Fig. 13 compares reduced basis method and FEM VSWR results with measurements carried out in [31]. Good agreement is obtained.

Since the electrical size of the UWB planar monopole is rather large in terms of wavelengths in the upper UWB band, the actual analysis domain, which should enclose the planar monopole with a sphere (see Fig. 12), is rather large too. Thus far, we may expect a large number of interior resonances in the upper UWB band, which, in turn, may increase the actual dimension of the reduced basis approximation as the band of interest for the reduced order model increases. Fig. 14 illustrates this situation.

\section{Conclusion}

A reduced basis method approach for reliable fast frequency sweep in the time-harmonic Maxwell's equations has been presented. This methodology is based on approximating the electromagnetic 


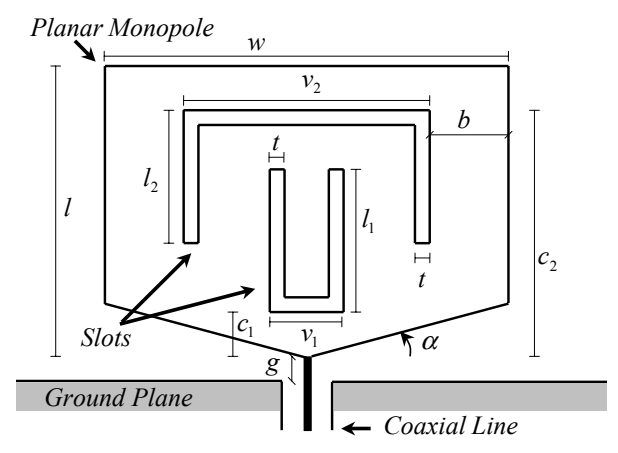

Figure 10: Geometry of the multiple band-notched planar monopole antenna: Side view. $l=$ $27 \mathrm{~mm}, w=20 \mathrm{~mm}, g=1 \mathrm{~mm}, \alpha=\pi / 15, b=3.75 \mathrm{~mm}, t=1 \mathrm{~mm}, c_{1}=1 \mathrm{~mm}, l_{1}=14.6 \mathrm{~mm}$, $v_{1}=5 \mathrm{~mm}, c_{2}=24.7 \mathrm{~mm}, l_{2}=19.35 \mathrm{~mm}, v_{2}=12.5 \mathrm{~mm}$.

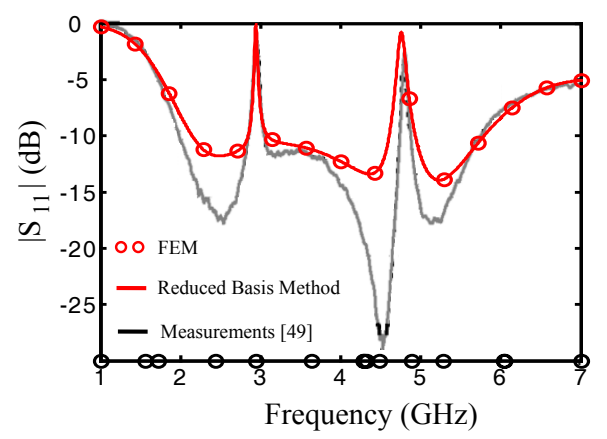

Figure 11: Reflection coefficient comparison between the proposed approach and measurements in the multiple band-notched planar monopole.

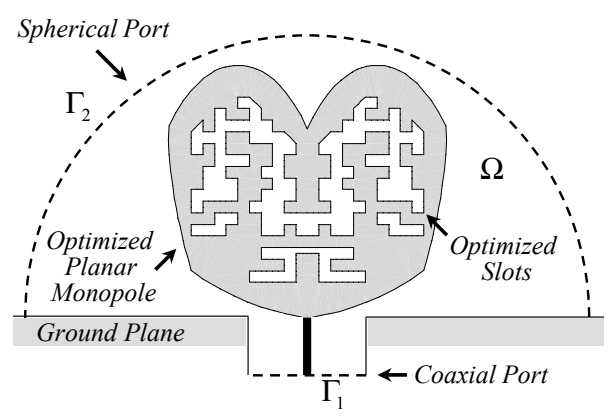

Figure 12: Geometry of the optimized band-notched UWB planar monopole antenna. 


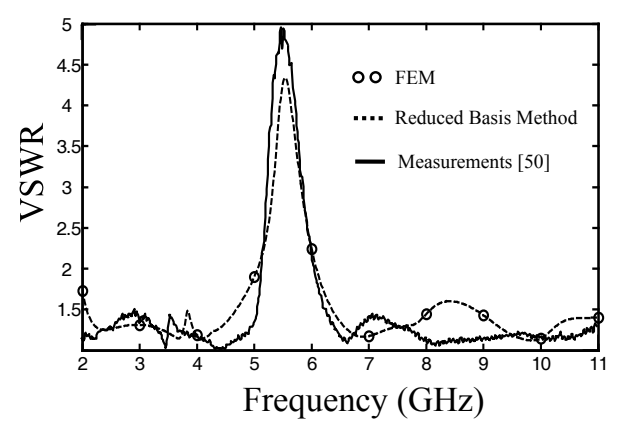

Figure 13: Comparison of the VSWR results between the proposed approach and measurements in the band-notched UWB planar monopole antenna.

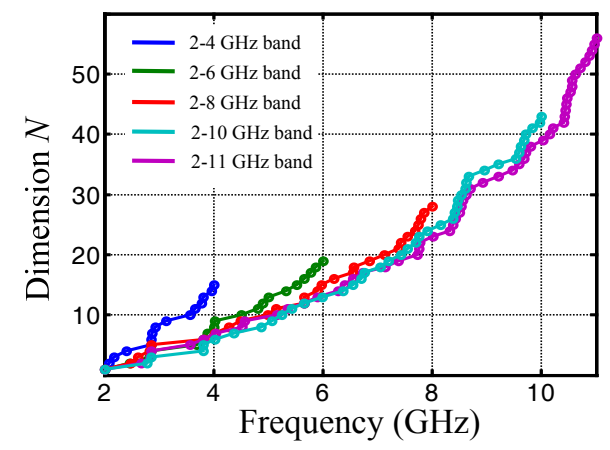

Figure 14: Reduced basis frequency sample points for different bands of interest in the bandnotched UWB planar monopole antenna. 
field as a member in the evolution of the electromagnetic field as frequency changes, rather than an arbitrary member in the field solution space. Crucial aspects from the computational point of view have been addressed. An offline-online decomposition strategy has been carried out to completely decouple those time-consuming parts from the many queries frequency evaluation stage. Special emphasis has been placed on an easily-computable error measure that actually serves to certify whether a frequency response via the reduced order model is accurate enough. As a result, a completely reliable model order reduction process has been presented. Based on these ingredients, a fast frequency sweep algorithm that automatically exits as soon as an accurate response is obtained throughout the large frequency band of interest has been detailed. Note that the band of interest should avoid the resonances in the current implementation of the method, but we refer to [10], [51] for improvements that allow to incorporate more closely these cases as well. Finally, some challenging microwave devices have shown the capabilities of the proposed approach.

\section{References}

[1] M. Ahmadloo and A. Dounavis. Parameterized model-order reduction for efficient eigenanalysis of dielectric waveguides structures. IEEE Trans. Microwave Theory Tech., 56(12):28512858, December 2008.

[2] B. O. Almrouth, P. Stern, and F. A. Brogan. Automatic choice of global shape functions in structural analysis. AIAA Journal, 16(5):525-528, May 1978.

[3] Z. Bai, R. D. Slone, W. T. Smith, and Q. Ye. Error bound for reduced system model by Padé approximation via the Lanczos process. IEEE Trans. Computer-Aided Design, 18(2):133-141, February 1999.

[4] Z. Bai and Q. Ye. Error estimation of the Padé approximation of transfer function via the Lanczos process. Elec. Trans. Numer. Anal., 7:1-17, 1998.

[5] M. Barrault, N. C. Nguyen, Y. Maday, and A. T. Patera. An empirical interpolation method: application to efficient reduced-basis discretization of partial differential equations. $C . R$. Acad. Sci. Paris, Ser. I, 339:667-672, 2004.

[6] A. Barret and G. Reddien. On the reduced basis method. Z. Angew. Math. Mech., 75(7):543-549, July 1995.

[7] F. Bertazzi, O. A. Peverini, M. Goano, G. Ghione, R. Orta, and R. Tascone. A fast reducedorder model for the full-wave FEM analysis of lossy inhomogeneous anisotropic waveguides. IEEE Trans. Microwave Theory Tech., 50(9):2108-2114, September 2002. 
[8] J. R. Brauer and G. C. Lizalek. Microwave filter analysis using a new 3-D finite-element modal frequency method. IEEE Trans. Microwave Theory Tech., 45(5):810-818, May 1997.

[9] H. Brezis. Analyse fonctionnelle. Théorie et applications. Masson, Paris, 1983.

[10] Y. chen, J. S. Hesthaven, Y. Maday, and J. Rodríguez. Improved succesive constraint method based a posteriori error estimate for reduced basis approximation of 2D Maxwell's problem. Elsevier Science, Submitted.

[11] V. de la Rubia and J. Zapata. MAM-a multipurpose admittance matrix for antenna design via the finite element method. IEEE Trans. Antennas Propagat., 55(8):2276-2286, August 2007.

[12] V. de la Rubia and J. Zapata. Microwave circuit design by means of direct decomposition in the finite-element method. IEEE Trans. Microwave Theory Tech., 55(7):1520-1530, July 2007.

[13] O. Farle, V. Hill, P. Ingelström, and R. Dyczij-Edlinger. Multi-parameter polynomial order reduction of linear finite element models. Math. Comput. Model. Dyn. Syst., 14(5):421-434, October 2008.

[14] O. Farle, V. Hill, P. Nickel, and R. Dyczij-Edlinger. Multivariate finite element model order reduction for permittivity or permeability estimation. IEEE Trans. Magn., 42(4):623-626, April 2006.

[15] P. Feldmann and R. W. Freund. Efficient linear circuit analysis by Padé approximation via the Lanczos process. IEEE Trans. Computer-Aided Design, 14(5):639-649, May 1995.

[16] V. Girault and P.-A. Raviart. Finite Element Methods for Navier-Stokes Equations. Springer-Verlag, Berlin, 1986.

[17] M. A. Grepl, Y. Maday, N. C. Nguyen, and A. T. Patera. Efficient reduced-basis treatment of nonaffine and nonlinear partial differential equations. M2AN Math. Model. Numer. Anal., 41(3):575-605, 2007.

[18] D. B. P. Huynh, G. Rozza, S. Sen, and A. T. Patera. A succesive constraint linear optimization method for lower bounds of parametric coercivity and inf-sup stability constants. C. R. Acad. Sci. Paris, Ser. I, 345:473-478, 2007.

[19] J. M. Jin. The Finite Element Method in Electromagnetics. Wiley-IEEE Press, New York, NY, second edition, 2002.

[20] A. Kolmogoroff. Über die beste ännaherung von Funktionen einer gegebenen Funktionenklasse. Anals of Maths., 37:107-110, 1963. 
[21] P. D. Ledger and K. Morgan. An adjoint enhanced reduced-order model for monostatic RCS computation. Electromagnetics, 28(1):54-76, 2008.

[22] P. D. Ledger, J. Peraire, K. Morgan, O. Hassan, and N. P. Weatherill. Parameterised electromagnetic scattering solutions for a range of incident wave angles. Comput. Meth. Appl. Mech. Eng., 193:3587-3605, 2004.

[23] S. H. Lee and J. M. Jin. Adaptive solution space projection for fast and robust wideband finite-element simulation of microwave components. IEEE Microwave Wireless Compon. Lett., 17(7):474-476, July 2007.

[24] S. H. Lee and J. M. Jin. Efficient full-wave analysis of multilayer interconnection structures using a novel domain decomposition-model-order reduction method. IEEE Trans. Microwave Theory Tech., 56(1):121-129, January 2008.

[25] W.-S. Lee, D.-Z Kim, K.-J. Kim, and J.-W. Yu. Wideband planar monopole antennas with dual band-notched characteristics. IEEE Trans. Microwave Theory Tech., 56(6):2800-2806, June 2006.

[26] L. Machiels, Y. Maday, I. B. Oliveira, A. T. Patera, and D. Rovas. Output bounds for reduced-basis approximations of symmetric positive definite eigenvalue problems. $C . R$. Acad. Sci. Paris, Ser. I, 331(2):153-158, 2000.

[27] Y. Maday. Reduced basis method for the rapid and reliable solution of partial differential equations. In Proceedings of the International Congress of Mathematicians. European Mathematical Society, 2006.

[28] Y. Maday, N. C. Nguyen, A. T. Patera, and G. S. H. Pau. A general multipurpose interpolation procedure: the magic points. Comm. Pure. Appl. Anal., 8(1):383-404, January 2009.

[29] Y. Maday, A. T. Patera, and D. V. Rovas. A blackbox reduced-basis output bound method for noncoercive linear problems. In D. Cioranescu and Jacques-Louis Lions, editors, Nonlinear Partial Differential Equations and Their Applications, pages 533-569. Elsevier Science, 2002.

[30] Y. Maday and U. Razafison. A reduced basis method applied to restricted Hartree-Fock equations. C. R. Acad. Sci. Paris, Ser. I, 336:243-248, 2008.

[31] J. Martínez-Fernández, V. de la Rubia, J. M. Gil, and J. Zapata. Frequency notched UWB planar monopole antenna optimization using a finite element method-based approach. IEEE Trans. Antennas Propagat., 56(9):2884-2893, September 2008. 
[32] J. Martínez-Fernández, J. M. Gil, and J. Zapata. Optimization of the profile of a planar ultra wide band monopole antenna in order to minimize return losses. In 2nd Eur. Conf. on Antennas and Propagation (EuCAP), November 11-16, 2007.

[33] J. Martínez-Fernández, J. M. Gil, and J. Zapata. Ultrawideband optimized profile monopole antenna by means of simulated annealing algorithm and the finite element method. IEEE Trans. Antennas Propagat., 55(6):1826-1832, June 2007.

[34] J. R. Montejo-Garai and J. Zapata. Full-wave design and realization of multicoupled dualmode circular waveguide filters. IEEE Trans. Microwave Theory Tech., 43(6):1290-1297, June 1995.

[35] J.-C. Nédélec. Mixed finite elements in $\mathrm{R}^{3}$. Numer. Math., 35:315-341, 1980.

[36] N. C. Nguyen and J. Peraire. An efficient reduced-order modeling approach for non-linear parametrized partial differential equations. Int. J. Numer. Meth. Eng., 56:27-55, 2008.

[37] N. C. Nguyen, K. Veroy, and A. T. Patera. Certified real-time solution of parametrized partial differential equations. In S. Yip, editor, Handbook of Materials Modelings, pages 1523-1558. Springer, 2005.

[38] A. K. Noor and J. M. Peters. Reduced basis technique for nonlinear analysis of structures. AIAA Journal, 18(4):455-462, April 1980.

[39] L. T. Pillage and R. A. Rohrer. Asymptotic waveform evaluation for timing analysis. IEEE Trans. Computer-Aided Design, 9(4):352-366, April 1990.

[40] V. V. S. Prakash, J. Yeo, and R. Mittra. An adaptive algorithm for fast frequency response computation of planar microwave structures. IEEE Trans. Microwave Theory Tech., 52(3):920-926, March 2004.

[41] J. Rubio, J. Arroyo, and J. Zapata. Analysis of passive microwave circuits by using a hybrid 2-D and 3-D finite-element mode-matching method. IEEE Trans. Microwave Theory Tech., 47(9):1746-1749, September 1999.

[42] J. Rubio, J. Arroyo, and J. Zapata. SFELP-an efficient methodology for microwave circuit analysis. IEEE Trans. Microwave Theory Tech., 49(3):509-516, March 2001.

[43] S. Sen, K. Veroy, D. B. P. Huynh, S. Deparis, N. C. Nguyen, and A. T. Patera. Natural norm a posterior error estimator for reduced basis approximations. J. Comput. Phys., $217(1): 37-62,2006$. 
[44] R. D. Slone, J.-F. Lee, and R. Lee. Multipoint Galerkin asymptotic waveform evaluation for model order reduction of frequency domain FEM electromagnetic radiation problems. IEEE Trans. Antennas Propagat., 49(10):1504-1513, October 2001.

[45] R. D. Slone, J.-F. Lee, and R. Lee. Automatic multipoint Galerkin AWE for a FEM fast frequency sweep. IEEE Trans. Magn., 38(2):637-640, March 2002.

[46] R. D. Slone, R. Lee, and J.-F. Lee. Well-conditioned asymptotic waveform evaluation for finite elements. IEEE Trans. Antennas Propagat., 51(9):2442-2446, September 2003.

[47] B. Stupfel. Frequency interpolation of the electromagnetic surface currents via singular value decomposition. application to high-frequency analysis. IEEE Trans. Antennas Propagat., 56(12):3789-3796, December 2008.

[48] D.-K Sun, J.-F. Lee, and Z. Cendes. ALPS-a new fast frequency-sweep procedure for microwave devices. IEEE Trans. Microwave Theory Tech., 49(2):398-402, February 2001.

[49] K. Veroy, C. Prud'homme, D. V. Rovas, and A. T. Patera. A posteriori error bounds for reduced-basis approximation of parametrized noncoercive and nonlinear elliptic partial differential equations. In Proc. AIAA Computational Fluid Dynamics Conference, 2003.

[50] J. P. Webb. Hierarchal vector basis functions of arbitrary order for triangular and tetrahedral finite elements. IEEE Trans. Antennas Propagat., 47(8):1244-1253, August 1999.

[51] Y. Maday Y. Chen, J. S. Hesthaven and J. Rodriguez. Certified reduced basis methods and output bounds for the harmonic Maxwell's equations. [Online]. Available:http://www.ann.jussieu.fr/publication/2009/R09014.pdf.

Valentín de la Rubia is with the Departamento de Tecnología de los Computadores y de las Communicaciones, Escuela Politécnica de Cáceres, Universidad de Extremadura, 10071 Cáceres, Spain, (e-mail: valentin.delarubia@upm.es.)

Ulrich Razafison is with the Université d'Orléans, UMR 6628, Laboratoire MAPMO, Fédération Denis Poisson, 45067, Orléans Cedex 2, France, (e-mail: ulrich.razafison@math.cnrs.fr).

Yvon Maday is with the Université Pierre et Marie Curie-Paris 6, UMR 7598, Laboratoire Jacques-Louis Lions, F-75005, Paris, France; and Division of Applied Mathematics, Brown University, USA, (e-mail: maday@ann.jussieu.fr) 